Prospective Evaluation

\title{
Impact of Urine Drug Screening on No Shows and Dropouts among Chronic Pain Patients: A Propensity-Matched Cohort Study
}

Parthasarathy Krishnamurthy, PhD ${ }^{1,2}$, Govindaraj Ranganathan, MD2, Courtney Williams, MD2, and Gulshan Doulatram, MD²

From: ' ${ }^{-U n i v e r s i t y ~ o f ~ H o u s t o n, ~}$ Houston, TX; ${ }^{2}$ University of Texas Medical Branch, Galveston, TX

Address Correspondence: Parthasarathy Krishnamurthy, PhD Dept. of Anesthesiology and Pain

Medicine

University of Texas Medical Branch

Dept. of Marketing and Entrepreneurship University of Houston 4800 Calhoun Houston, TX 77004 E-mail:

partha@uh.edu

Disclaimer: There was no external funding in the preparation of this manuscript.

Conflict of interest: Each author certifies that he or she, or a member of his or her immediate family, has no commercia association (i.e., consultancies, stock ownership, equity interest, patent/licensing arrangements, etc.) that might pose a conflict of interest in connection with the submitted manuscript.

Manuscript received: 04-01-015 Revised manuscript received: o6-24-2015 Accepted for publication: 07-02-2015

Free full manuscript: www.painphysicianjournal.com
Background: The last 2 decades have seen a substantial increase in both the prescription of opioids for managing chronic pain, and an increase in opioid-related deaths in the US. Urine drug screening (UDS) is the de facto monitoring tool aimed at detecting and deterring opioid misuse.

Objective: We study whether administering UDS on pain patients influences post-screening behavior of no-shows and dropouts.

Study Design: Observational cohort study of electronic medical records.

Setting: Single urban academic pain-clinic.

Methods: A retrospective cohort comparison of patients receiving UDS versus those not receiving UDS was conducted on the entire sample as well as in the propensity score-matched samples in which matching was based on age, gender, pain-score, procedure-scheduled, systolic and diastolic blood pressure (BP), pulse, temperature, physician ID, year of visit, psychology referral, and opioid prescription in the first visit. In addition, we conducted within-subjects logistic-regression to study no-shows and non-proportional hazards survival modeling to study dropout.

Results: Analyses of 4,448 clinic visits by 723 pain patients indicated that UDS exposure in the first visit is associated with increased risk of no-show in the second visit (OR $=2.73, P<$ .0001); no-show rate was $10.24 \%$ for those without UDS compared to $23.75 \%$ for those with a UDS. Among those tested, the no-show rate was higher for those testing positive for illicit substances (34.57\%) than for those testing negative $(21.74 \%)$. These findings were replicated in 8 different propensity-score matched subsamples aimed at addressing potential nonrandom selection, as well as in within-subject analysis accounting for individual-level no-show propensity. Non-proportional hazards survival analysis shows that risk of dropout increased by $100.3 \%$ with every additional UDS (HR 95\% Cl: 1.54 to 2.61 ).

Limitations: Retrospective design, non-randomized sample, single-setting.

Conclusions: The results indicate that UDS is associated with increased no-shows and dropout from clinic subject to limitations of observational studies such as selection bias and confound by unobserved variables. These results serve as a call for additional prospective randomized studies to understand the impact of UDS, and where the patients might go when they dropout from the clinic.

Key words: Chronic pain, opioid monitoring, UDS, urine-drug screening, no-show, dropout, adherence, propensity-score matching

Pain Physician 2016; 19:89-100 
P ain management is a critical element of patient care. Over the last 2 decades the emphasis on managing pain has led to a substantial increase in the prescription of opioids $(1,2)$. While opioids can significantly improve the quality of life for the patients, there are many concerns. Some are biological, and include opioid tolerance, opioid-induced abnormal pain sensitivity, hormonal consequences, and abnormal immune modulation. Equally of concern are the behavioral issues of abuse (3) (overdose (4) is the cause of 20,000 deaths per year), diversion, and recreational use $(5,6)$. Therefore, monitoring adherence for patients on (or considered candidates for) opioid treatment is a critical element of pain management.

To this end, patients and physicians jointly execute monitoring agreements (opioid contracts) which describe the outcome expectations of opioid therapy and the behavioral expectations of the patient. The latter include agreeing to urine drug screening (UDS), filling prescriptions in the same pharmacy when possible, making pill counts possible, avoiding illicit substances, adhering to prescribed dosage, not sharing medications, and providing self-reports $(3,7)$ as noted by American Pain Society and the American Academy of Pain Medicine (8). Of the various tools, UDS is perhaps the most effective in detecting non-adherence, and is viewed as the de facto monitoring tool $(2,5,8,9)$.

Although UDS is widely accepted as an important element of pain management, despite the challenges raised by UDS (10), there is relatively little research on how it influences adherence behaviors. We focus on whether administering UDS results in patients keeping appointments and not discontinuing therapy unannounced (dropout). Prior research specifies a wide set of behaviors as aberrant in the context of opioid therapy $(3,11)$, including diversion, consumption of illicit substances, dose escalations, pre-mature requests for refills, visiting multiple providers, failure to keep appointment (no-show), and discontinuation from therapy (dropout).

Of these, no-shows and dropouts are particularly important from an adherence monitoring perspective because they are visit-independent indices of aberrant behaviors. In contrast, monitoring of other aberrant behaviors like diversion, dose escalation, failure to consume prescribed opioids, and consumption of illicit substances can be done only when there is a patientvisit. As a result, visit-dependent indices of adherence censor the observations to those who have chosen to come to the clinic. Such patients are more likely to be adherent than non-adherent.. Therefore, studying visit-dependent indices of adherence without accounting for no-show and dropout may present a more favorable picture of the impact of UDS on adherence than what the true state of adherence might be. Second, dropouts and no-shows represent patients who potentially begin to disengage from a given clinic. Although the observation of disengagement does not tell why and where the patients are going, given findings in the literature that exposure to prescription opioids sometimes serve as gateway drugs to street drugs like heroin (12), there is a risk that some of the patients dropping out may be heading for the street. For these reasons, we believe that it is important to study no-shows and dropouts.

\section{Literature on Urine Drug Screening}

A comprehensive description of the literature on UDS in opioid management is beyond the scope of this manuscript (see Chou et al (8) and Starrels et al (13) for reviews). However, the research on UDS appears to center on 2 broad themes: its efficacy as a tool for detecting opioid-related misuse, and its consequences for patient behavior. Given the focus of our research, we discuss the latter.

In regards to patient attitudes and behavior, the literature examines a variety of questions including predicting abuse, impact of testing on post-testing behavior, and to intended/unintended consequences of testing. In regards to predictors of abuse, the findings are equivocal. While some studies implicate psychological factors (14) and self-reported past history of drug abuse (3) as potential predictors of abuse, reviews of several studies find no robust evidence of a set of predictors that predict abuse among chronic pain patients $(15,16)$.

We found a handful of papers on the effect of UDS on post-screening behavior. One study suggests that random drug screening may be associated with a reduction in prevalence of marijuana in urine screens (7). This study compared the proportion of patients testing positive for illicit substances before and after a UDS regime was put in place, and reported that the proportion was lower in the "after" group. However, as noted by Chou et al (16), this study presents non-statistical comparison of 2 historical cohorts that did not overlap in time that constrains the interpretability of the findings. Overall, Chou et al's (16) review finds no noticeable impact of a variety of monitoring tools (UDS, pill-counts, prescription monitoring programs) on patient behavior/ outcomes. A similar view is espoused in another review by Starrels et al (13), which focuses specifically on the 


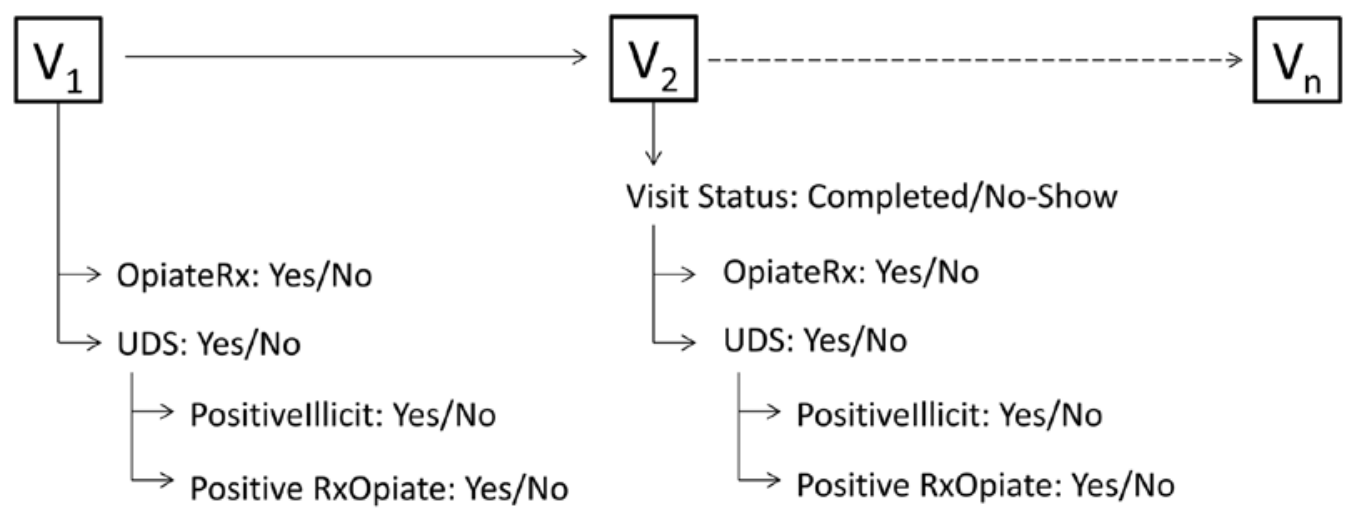

Fig. 1. Simplified timeline of clinical visits and events.

Fig. 1 represents a simplified/stylized timeline of clinic events, V1-Vn, for a patient over his/her tenure. In each visit, the patient may show up $(\mathrm{Y} / \mathrm{N})$, and if they show up, may receive prescription opioids, may receive a UDS, and if tested, may test positive or negative for illicit substances and positive/negative for prescribed opioids. This allows us to test whether UDS in any given visit influences no-show in subsequent visit. In addition, as of the last-visit, Vn, the patient's status can be either "continuing," "discharged," or "dropped-out" (not depicted in figure), and whether the cumulative number of UDS in a patient's tenure influences dropout.

impact of treatment contracts and UDS on opioid abuse.

Separate from predicting abuse and influencing adherence, there is also the concern that testing may have some adverse consequences. Katz and Fanciullo (17) suggest that subjecting patients to monitoring has the potential to injure the therapeutic alliance, cause patients to feel that they are not being trusted, or that they are being singled-out, besides raising patients' concerns about privacy of the results. This is an important concern as it refers to potential unintended effects of UDS.

In sum, while there is evidence indicating that UDS can be effective in detecting aberrant behaviors, its influence on patient behavior following the screening is not well-studied. To help situate our research question, consider the following simplified time-line of events in the tenure of a chronic pain patient (Fig. 1).

In the first visit $\left(V_{1}\right)$, the patient may or may not receive prescription opioids. Furthermore, the patient may or may not receive a UDS, and if tested, may test positive or negative for illicit substances, and test positive/negative for prescribed opioids. The appointment for the second visit $\left(V_{2}\right)$ is set at the completion of $V_{1}$, but before either the physician or patient is aware of the results of UDS in $V_{1}$. In the second visit, the patient may keep the appointment or be a no-show. Furthermore, if the patient does indeed keep the appointment, $\mathrm{V}_{2}$ may include an opioid prescription, a UDS or not, and if so, the patient may test positive or negative for illicit substances, and test positive or negative for prescribed opioids. Notice that $V_{2}$ can represent any subsequent visit, until the last visit, $V_{n}$. This simplified timeline allows us to raise the following questions regarding the relationship between UDS and no-shows and dropouts:

1. Is there a relationship between UDS in the first visit $V_{1}$ and the likelihood that the patient will keep the appointment in the second visit $\mathrm{V}_{2}$ ? More generally, is UDS in any visit associated with no-show in the subsequent visit?

2. Is there a relationship between the outcome of $V_{1}$ UDS (testing positive or negative for illicit substances) and the likelihood of keeping the appointment in $\mathrm{V}_{2}$ ?

3. Is there a relationship between the cumulative number of UDS a person receives in their tenure and the likelihood of unannounced dropout from the clinic?

\section{Methods}

\section{Ethics Statement}

The protocol has received ethics approval by the Committee for the Protection of Human Subjects from the University of Houston and the University of Texas, Medical Branch. 


\section{Data Collection}

The data for the analysis came from a cohort of patients in a university-based pain clinic. The dataset consisted of de-identified electronic medical records of 4,448 clinic encounters from 723 chronic pain patients who were admitted into practice starting April 9, 2009, through January 31, 2012, and having had at least 2 clinic encounters (Fig. 2 provides flow diagram of the set of analyzed patients was derived from the set of eligible patients).

The dataset includes details of each encounter including date of encounter, type of encounter (nurse visit and office visit - we excluded encounters that were phone calls or refill requests), the physician ID, results of the encounter (completed, no-show, canceled), vitals (pain score, systolic and diastolic blood pressure (BP), pulse, temperature), physician orders (including prescription for opioids, non-opioids, UDS, and other laboratory tests), results of UDS testing (positive/negative for illicit substances, and positive/negative for prescribed opioids), referral for psychological counseling, and notes summary in which planned pain-interven- tional procedures were documented. In addition, information about age and gender was available in the dataset.

\section{Measures of Principal Variables}

The electronic medical records at the individual-patient-encounter level allow us to construct a timeline of various clinic-related events over the tenure of the patient including when UDS took place if it did, the results of the testing, whether the patient kept subsequent appointments, and whether they dropped out.

The 2 principal dependent variables are the following events: the status of the second appointment and unannounced dropout from therapy. To study impact of UDS on no-shows, we examined whether UDS in $V_{1}$ was related to no-show in $\mathrm{V}_{2}$ (see Fig. 1), and whether it was related to the results of the UDS. No-show in the second appointment is readily measured because the second appointment is scheduled at the end of the office visit with the patient. Since assignment to UDS in first visit is not random, we employed propensity score matching to test the association between UDS and no-

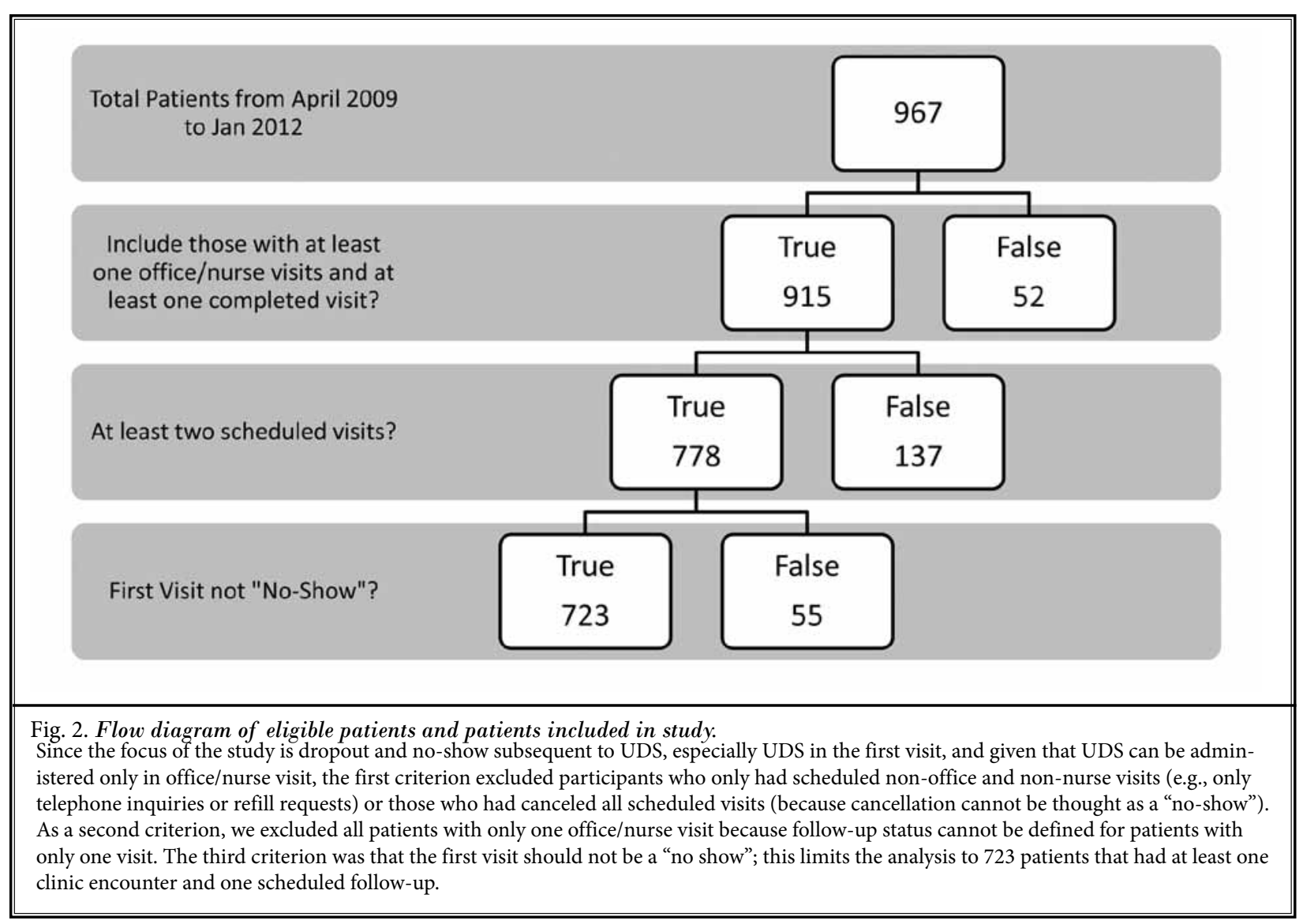


show in the second visit. This is described in greater detail in the next section.

In regards to dropout, since there is no direct measure of dropout, we considered a patient to have dropped-out if the most recent appointment was a noshow and the duration since the last contact was greater than 3-times the average inter-visit duration for that patient. For instance, if a patient visits the clinic every 45 days, and the patient has not contacted the clinic in 135 days and the last scheduled visit was a no-show, we considered this patient to have dropout-out without announcement. We refer to this measure of dropout as "dropout3x." To test the robustness of our results, we specified 2 additional definitions of dropout, "dropout180" defined as 180 days since last no-show, and "dropout365" defined as 365 days since last no-show.

\section{Statistical Analysis}

\section{Assessing Effect of $V_{1}$ UDS on $V_{2}$ No-show}

Although every patient prior to visit received a letter indicating that the practice may monitor adherence, the administration of UDS (which came toward the end of the visit) was not necessarily random. For this reason, we employed propensity score matching to adjust for potential selection and confounding as is traditionally done in observational studies (18).

First, we used logistical regression using all available variables as predictors of the exposure to treatment (in our case, UDS in first visit) to generate the propensity for receiving the treatment. There were 11 available covariates as of the first visit. This included 2 demographic variables: gender and age, and $9 \mathrm{~V}_{1}$-specific variables: pain score, pulse, systolic BP, diastolic BP, physician ID, year of visit, whether there was an opioid prescription in the visit, whether a pain-interventional procedure was planned for $\mathrm{V}_{2}$, and whether there was a psychological referral in the visit.

Second, we generated the propensity-score matched datasets using 2 different variations of the nearest-neighbor algorithm, the greedy-match algorithm (PS-Greedy) which maximizes the odds of a match and a caliper-based match algorithm (PS-Caliper) which minimizes the odds of a mismatch and is reported to produce estimates with lower bias (19). This resulted in 3 different datasets to study the effect of UDS in $V_{1}$ on no-show in $\mathrm{V}_{2}$, the baseline full dataset, the PS-matched subsample based on the greedy-match algorithm, and the PS-matched subsample based on the caliper-based nearest neighbor algorithm. We generated the PS- matched subsamples using the \%PSMACRO written for the SAS system $(20,21)$, specifying $1: 1$ selection without replacement, and setting the caliper size as 0.3 standard deviation of propensity score for the caliper-based matching subsample.

Third, the central research question was assessed by specifying a logistic regression model with exposure to UDS in $\mathrm{V}_{1}$ as the predictor of no-show in $\mathrm{V}_{2}$. The 10 variations of this basic model came from the variations in the dataset and the covariates specified in the model. In the baseline (full) dataset we specified 2 models: the unadjusted model and the all covariates adjusted model. In each of the 2 PS-matched subsamples, we specified 4 different models: the unadjusted model, the propensity-score adjusted model, the propensityscore and significant covariates adjusted model, and the propensity-score and all covariates adjusted model.

\section{Assessing the Effect of Cumulative UDS on Dropout}

In regards to dropout, we employed a non-proportional hazards survival model to assess the effect of UDS on dropout. Specifically we modeled duration to dropout with status of dropout (yes $=1 /$ no $=0$ ) as the censoring variable, using cumulative number of UDS as a time-varying predictor, and current pain-level, cumulative number of psychological counseling, cumulative number of opioid prescriptions as additional time-varying control variables, and age and gender as static control variables. We performed additional tests of robustness described in the results section.

\section{Descriptive Results}

The sample comprised 723 patients (64\% female) with 4,448 unique face-to-face patient-encounters, with each patient with at least 2 scheduled visits and one completed visit. The descriptive results are presented in Table 1.

\section{Difference between UDS and No UDS Patients}

We first specified a logistic regression model with the 11 covariates previously mentioned to assess the differences between the UDS and No UDS sample, and generate the propensity score for receiving UDS. The propensity scores for the likelihood of receiving UDS using all covariates available as of the first visit ranged from 0.03 to 0.99 .

We observed significant differences in several of the covariates between the UDS and No UDS groups (Table 2); the likelihood of receiving UDS in the first 
Table 1. Descriptive statistics.

\begin{tabular}{|l|c|c|}
\hline Sample Descriptors & 723 \\
\hline Number of Patients & 4,448 \\
\hline Number of Contacts & $65 \%$ Female \\
\hline Gender $\quad$ Individual-Level Descriptors & $21 \%$ & \multicolumn{2}{|c|}{ Median } \\
\hline \% Dropping Out & 50.9 & 51 \\
\hline Age & 475.8 & 505 \\
\hline Observed Duration & 72.2 & 350 \\
\hline Inter-visit Duration & 406.4 & 4 \\
\hline Days to Dropout & 6.1 & 1 \\
\hline Number of Contacts & 1.2 & 0 \\
\hline Number of UDS & 0.7 & 2 \\
\hline Number of No shows & 3.3 & 0 \\
\hline Number of Opioid Rx & 0.2 & 1 \\
\hline Number of +ve test for Illicit Substances & 0.6 & \\
\hline Number of +ve tests for Rxed Opioids & & \\
\hline
\end{tabular}

visit was influenced by diastolic BP $(P<.0068)$, systolic BP $(P<.0955)$, calendar year of visit $(P<.0001)$, whether there was a pain procedure scheduled for $\mathrm{V}_{2}(P<.0127)$, whether there was an opioid prescription in the visit $(P$ $<.0031)$, and whether there was a psychology referral $(P<$.0994). To address these differences, we generated the propensity-score adjusted subsamples based on the greedy algorithm and the caliper-match algorithm as specified earlier. The results of the match are described below.

\section{PS-Greedy Match Sub-sample}

In the greedy match sub-sample, many of the differences between the UDS and No-UDS sample (Table 2 ) were noticeably reduced (Table 4). However, diastolic BP $(P<.0639)$, calendar year of visit $(P<.0001)$, and whether there was a pain procedure scheduled for the next visit $(P<.0881)$ remained significant predictors of UDS in the first visit. This suggested that the greedymatch algorithm was partially successful in reducing the imbalance.

\section{PS-Caliper Match Sub-sample}

In the caliper-based match sub-sample, many of the differences between the UDS and No-UDS sample (seen in Table 2) were absent (Table 4). Only the calendar year of visit $(P<.1)$ remained as a marginally significant pre- dictor of UDS in the first visit. This suggested that the caliper-based match algorithm was mostly successful in reducing the imbalance.

\section{Impact of $V_{1}$ UDS on $V_{2}$ No-Show}

We now turn to the results pertaining to the principal research question: the effect of UDS in first visit on no-show in the second visit for all the models in the 3 samples described below and summarized in Table 5 .

\section{Full Sample}

We specified 2 models in the full sample, the unadjusted and all-covariates adjusted model. As seen in Table 5, UDS in the first visit was associated with significantly higher rate of no-show in the second visit, both in the unadjusted as well as in the all-covariates adjusted model (odds ratio $[O R]=2.73,95 \% \mathrm{Cl} 1.66-4.47$, $P<.0001$, in the adjusted model, and OR $=2.18,95 \%$ $\mathrm{Cl} 1.22-3.88, P<.0084$, in the all-covariates adjusted model).

\section{Greedy-Match Subsample}

As shown in Table 5, UDS in the first visit was associated with significantly higher rate of no-show in the second visit in all 4 models (OR $=3.29,95 \% \mathrm{Cl} 1.91$ $-5.69, P<.0001$, in the unadjusted model, OR $=2.79$, $95 \% \mathrm{Cl} 1.45-5.36, P<.0021$, in the propensity score ad- 
Impact of Urine Drug Screening on No Shows and Dropouts among Pain Patients

Table 2. Characteristics of patients with UDS and no UDS in first visit. (Full sample $n=723$ )

\begin{tabular}{|l|c|c|c|c||}
\hline & No UDS & UDS & ChiSq & P-Value \\
\hline Gender (\% Female) & 64.88 & 62.93 & 0.72 & 0.3949 \\
\hline Age (years) & 52.12 & 49.49 & 2.30 & 0.1296 \\
\hline Pain Score & 6.62 & 6.94 & 0.24 & 0.6217 \\
\hline Pulse (bpm) & 79.90 & 82.14 & 0.09 & 0.7606 \\
\hline Diastolic BP & 80.78 & 83.72 & 7.34 & 0.0068 \\
\hline Systolic BP & 133.98 & 134.35 & 2.78 & 0.0955 \\
\hline VisitProvider ID & & & 16.97 & 0.1509 \\
\hline Start Year & & & 101.60 & $<.0001$ \\
\hline 2009 (\%) & 44.39 & 6.95 & & \\
\hline 2010 (\%) & 32.68 & 40.35 & & \\
\hline 2011 (\%) & 22.93 & 52.70 & & \\
\hline Procedure Scheduled & & & 6.21 & 0.0127 \\
\hline Yes (\%) & 37.56 & 32.24 & & \\
\hline Opioid Rx in Visit 1 & & & 8.75 & 0.0031 \\
\hline Yes (\%) & 38.54 & 40.73 & & \\
\hline Psychology Ref in Visit 1 & & & 2.72 & 0.0994 \\
\hline Yes (\%) & 10.24 & 22.01 & & \\
\hline \hline
\end{tabular}

All chi-squared values are from the logistic regression in which all covariates predict likelihood of receiving UDS in first visit. The percentages are from the contingency table of UDS in visit $1(\mathrm{Yes} / \mathrm{No}) \times$ covariate levels. This table shows the UDS/No UDS imbalance in the full dataset. The propensity score generated from this model was used in the matching algorithms.

Table 4. Characteristics of patients with UDS and no UDS in first visit. (PS-Caliper Match Sub-Sample $n=318$ )

\begin{tabular}{|l|l|l|l|l||}
\hline & No UDS & UDS & ChiSq & P-Value \\
\hline Gender (\% Female) & 61.64 & 66.67 & 0.1509 & 0.6977 \\
\hline Age (years) & 50.86 & 50.65 & 0.1878 & 0.6647 \\
\hline Pain Score & 6.64 & 7.09 & 1.2586 & 0.2619 \\
\hline Pulse (bpm) & 80.77 & 80.96 & 0.0388 & 0.8438 \\
\hline Diastolic BP & 81.20 & 82.34 & 0.3666 & 0.5449 \\
\hline Systolic BP & 133.64 & 133.97 & 0.0893 & 0.7650 \\
\hline VisitProvider ID & & & 5.5515 & 0.9370 \\
\hline Start Year & & & 4.7460 & 0.0932 \\
\hline 2009 (\%) & 30.19 & 19.5 & & \\
\hline 2010 (\%) & 41.51 & 42.77 & & \\
\hline 2011 (\%) & 28.3 & 37.74 & & \\
\hline Procedure Scheduled & & & 0.1426 & 0.7057 \\
\hline Yes (\%) & 35.22 & 34.59 & & \\
\hline Opioid Rx in Visit 1 & & & 0.0633 & 0.8013 \\
\hline Yes (\%) & 40.88 & 40.88 & & \\
\hline Psychology Ref in Visit 1 & & & 1.5559 & 0.2123 \\
\hline Yes (\%) & 13.21 & 22.01 & & \\
\hline
\end{tabular}

This table shows the almost complete removal of imbalance in the PSCaliper match sub-sample compared to the full dataset (table 2).

www.painphysicianjournal.com
Table 3. Characteristics of patients with UDS and no UDS in first visit . (PS-Greedy Match Sub-Sample n = 410)

\begin{tabular}{|l|c|c|c|c|}
\hline & No UDS & UDS & ChiSq & P-Value \\
\hline Gender (\% Female) & 64.88 & 66.34 & 0.0634 & 0.8013 \\
\hline Age (years) & 52.12 & 50.15 & 1.055 & 0.3043 \\
\hline Pain Score & 6.62 & 6.86 & 0.0887 & 0.7658 \\
\hline Pulse (bpm) & 79.90 & 81.67 & 0.086 & 0.7693 \\
\hline Diastolic BP & 80.78 & 82.80 & 3.4341 & 0.0639 \\
\hline Systolic BP & 133.98 & 133.62 & 1.3936 & 0.2378 \\
\hline VisitProvider ID & & & 11.11 & 0.5193 \\
\hline Start Year & & & 60.98 & $<.0001$ \\
\hline 2009 (\%) & 44.39 & 5.37 & & \\
\hline 2010 (\%) & 32.68 & 39.51 & & \\
\hline 2011 (\%) & 22.93 & 55.12 & & \\
\hline Procedure Scheduled & & & 2.91 & 0.0881 \\
\hline Yes (\%) & 37.56 & 30.73 & & \\
\hline Opioid Rx in Visit 1 & & & 2.48 & 0.1151 \\
\hline Yes (\%) & 38.54 & 35.12 & & \\
\hline $\begin{array}{l}\text { Psychology Ref in } \\
\text { Visit 1 }\end{array}$ & 10.24 & 24.88 & & 0.2254 \\
\hline Yes (\%) & & & 1.47 & \\
\hline
\end{tabular}

This table shows some reduction in the imbalance in the PS-Greedy match sub-sample compared to the full dataset (Table 2).

justed model, OR $=2.84,95 \% \mathrm{Cl} 1.46-5.53, P<.0021$, in the propensity score and significant covariates adjusted model, and $\mathrm{OR}=3.12,95 \% \mathrm{Cl} 1.56-6.24, P<.0013$, in the all-covariates adjusted model).

\section{Caliper-Match Subsample}

As shown in Table 5, UDS in the first visit was associated with significantly higher rate of no-show in the second visit in all 4 models $(\mathrm{OR}=3.03,95 \% \mathrm{Cl} 1.56$ $-5.88, P<.0010$, in the unadjusted model, $\mathrm{OR}=2.46$, $95 \% \mathrm{Cl} 1.24-4.85, P<.0097$, in the propensity score adjusted model, $\mathrm{OR}=2.42,95 \% \mathrm{Cl} 1.23-4.79, P<.0110$, in the propensity score and significant covariates adjusted model, and $\mathrm{OR}=2.75,95 \% \mathrm{Cl} 1.30-5.81, P<0.0079$, in the all-covariates adjusted model).

\section{Impact of $V_{1}$ UDS on $V_{2}$ No-Show: Within- Patient Analysis}

In the within-patient analysis, we examined whether no-show in any given visit (from the second visit onward) is associated with the administration of UDS in the previous visit. Since this a within-patient analysis, 
we employed the Generalized Estimating Equations approach in which the patient's study identifier was specified as a repeated-subject or nesting factor, allowing us to control for unobserved patient-specific characteristics that might alter the results. UDS versus No-UDS had an odds-ratio of $1.51, P<.0001$, suggesting that those with UDS in the preceding visit had 1.51 times the odds of being a no-show in any given visit compared to those without UDS in the preceding visit. The results of the

Table 5. Effects of visit 1 UDS on no-show in visit 2 (Full Sample, PS-Greedy Sub-sample, PS-Caliper Sub-sample).

\begin{tabular}{|c|c|c|c|c|c|c|c|}
\hline & \multirow{2}{*}{$\mathbf{N}$} & \multirow{2}{*}{$\begin{array}{c}\text { No UDS } \\
(\%)\end{array}$} & \multirow{2}{*}{$\begin{array}{l}\text { UDS } \\
(\%)\end{array}$} & \multirow{2}{*}{$\mathbf{O R}$} & \multicolumn{2}{|c|}{$95 \%$ CI } & \multirow{2}{*}{$P$-value } \\
\hline & & & & & Lower & Upper & \\
\hline \multicolumn{8}{|l|}{ Full Sample } \\
\hline \multicolumn{8}{|l|}{ No-Show in Visit 2} \\
\hline Unadjusted & 723 & 10.24 & 23.75 & 2.73 & 1.66 & 4.47 & $<.0001$ \\
\hline All Cov Adjusted & 708 & 10.26 & 23.59 & 2.18 & 1.22 & 3.88 & $<.0084$ \\
\hline \multicolumn{8}{|c|}{ PS-Greedy Match Subsample } \\
\hline \multicolumn{8}{|c|}{ No-Show in Visit 2} \\
\hline Unadjusted & 410 & 10.24 & 27.32 & 3.29 & 1.91 & 5.69 & $<.0001$ \\
\hline PS Adjusted & 397 & 10.20 & 26.73 & 2.79 & 1.45 & 5.36 & $<.0021$ \\
\hline PS + Sig Covars ${ }^{*}$ & & & & 2.84 & 1.46 & 5.53 & $<.0021$ \\
\hline PS + All Covars ${ }^{\star}$ & & & & 3.12 & 1.56 & 6.24 & $<.0013$ \\
\hline \multicolumn{8}{|c|}{ PS-Caliper Match Subsample } \\
\hline \multicolumn{8}{|l|}{ No Show in Visit 2} \\
\hline Unadjusted & 318 & 8.81 & 22.64 & 3.03 & 1.56 & 5.88 & $<.0010$ \\
\hline PS Adjusted & 308 & 9.09 & 22.08 & 2.46 & 1.24 & 4.85 & $<.0097$ \\
\hline \multicolumn{3}{|l|}{ PS + Sig Covars* } & & 2.42 & 1.23 & 4.79 & $<.0110$ \\
\hline \multicolumn{3}{|l|}{ PS + All Covars ${ }^{*}$} & & 2.75 & 1.30 & 5.81 & $<.0079$ \\
\hline
\end{tabular}

In all the above models, we specified a logit link-function to generate the OR estimates above. However, in the 2 PS + All Covars Adjusted models we observed possible quasi-complete separation of the predicted variable based on the combination of predictors. In such instances we specified a probit link-function and found that the effects were consistent with the logit link-function results in terms of size, sign, and significance.

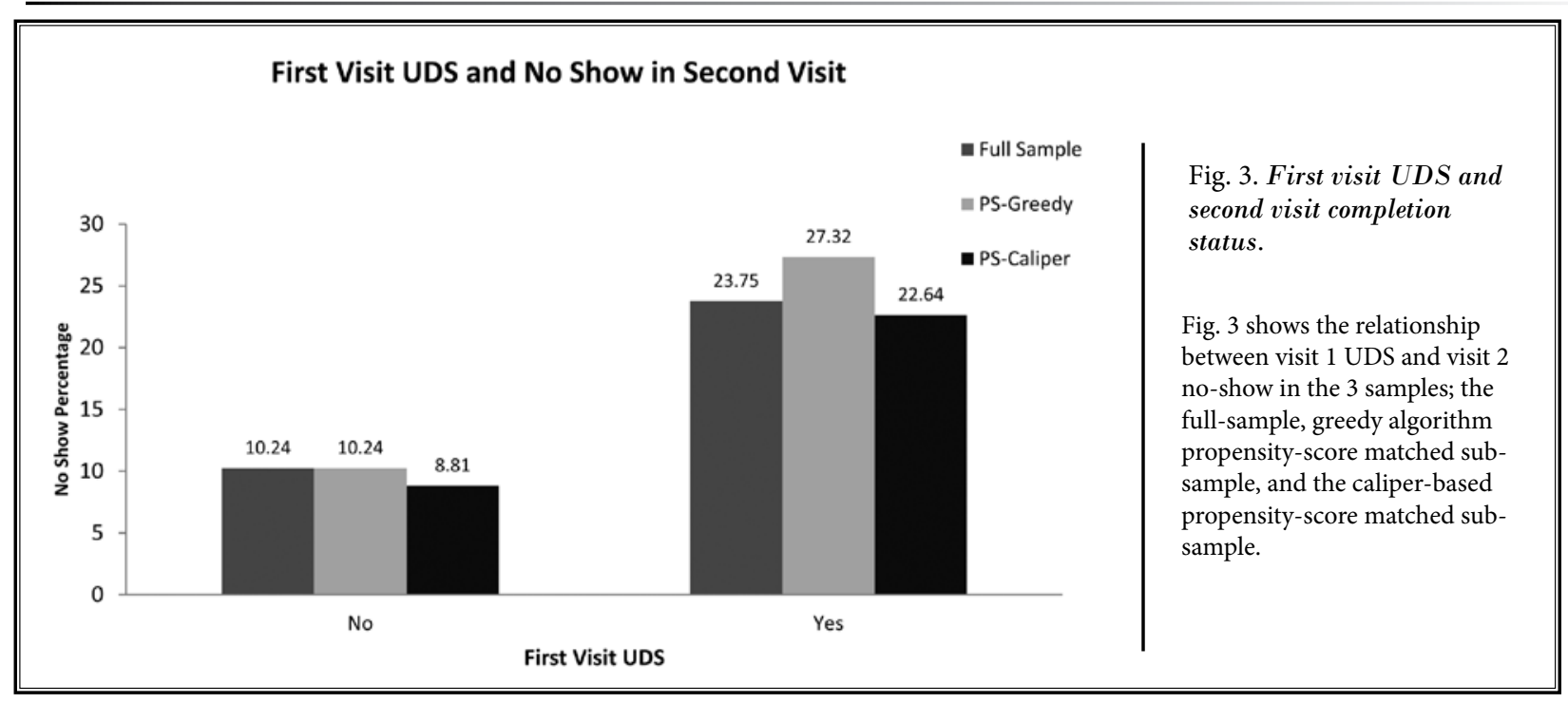


within-patient analysis are consistent with the baseline and propensity-score matched analysis.

In summary regardless of the analytical approach we took, propensity-score matching and within-patient longitudinal analysis, UDS appears to be associated with increasing the chance of no-shows in the subsequent visit.

\section{Impact of UDS Screening Results on No-Show in Subsequent Visit}

Our second question was whether the testing positive for illicit substances in $V_{1}$ UDS is associated with noshow in $\mathrm{V}_{2}$. We found this to be the case. Among the 518 people who received UDS in $\mathrm{V}_{1}$, those who tested positive for illicit substances were more likely to be noshows in $\mathrm{V}_{2}$ compared to those who tested negative, $35.44 \%$ versus $21.43 \%$. This was true both in the unadjusted model ( $\mathrm{n}=518, \mathrm{OR}=1.90,95 \% \mathrm{Cl}$ : 1.14 to $3.17, P$ $<.0137)$ as well as in the all-covariates adjusted model ( $n=513$, OR $=1.82,95 \% \mathrm{Cl}: 1.02$ to $3.22, P<.0417$ ).

It is noteworthy that even among those testing negative for illicit substances in $\mathrm{V}_{1}$, the no-show rate was significantly higher (21.74) compared to those not getting UDS in $\mathrm{V}_{1}(10.26), P<.0007$.

\section{Impact of UDS on Dropout from Therapy}

Our third question was whether UDS is related to dropout from therapy. We specified a non-proportional hazards survival-model with cumulative number of UDS, no-shows, psychological counseling references, and current pain as time-varying predictors, in addition to age and gender as static predictors. The time to dropout was the number of days from the first visit through dropout or end of observation (for censored observations). The results (Table 6 below) suggest that risk of dropout increased by $100.3 \%$ with every additional UDS (HR 95\% Cl: 1.53 to 2.61).

We specified several additional variations of the core model above to test the robustness of the results. We found similar results when dropout is defined using the 2 alternate measures of dropout180 and dropout365 (Table 6). The time-varying predictors in the core model are cumulative. For this reason, we defined the change in the cumulative predictors over the previous 120 days as an alternative non-cumulative time-varying covariate. The results were consistent: UDS increased risk of dropout (the detailed model results are available from the authors).

\section{Discussion}

Urine drug screening is a mainstay in informing physicians about potential aberrant behaviors on the part of patients on opioid medications. While there is considerable research on the value of UDS to the physician, there is little research on how it influences patient behavior.

The findings from the baseline and propensityscore matched datasets can be summarized broadly as follows: The post-screening behavior of those receiving a UDS was markedly different from those who did not.
Fig. 4. UDS outcome and second visit completion status.

Fig. 4 shows the relationship between whether UDS was positive for illicit substances $(\mathrm{Y} / \mathrm{N})$ and the proportion of patients that failed to keep the second scheduled clinic appointment. Notice that the no-show rate for those testing negative $(21.74 \%)$ is higher than the no-show rate for those not tested $(10.24 \%$, from Fig. 1).

\section{Result of First Visit UDS and No Show in Second Visit}

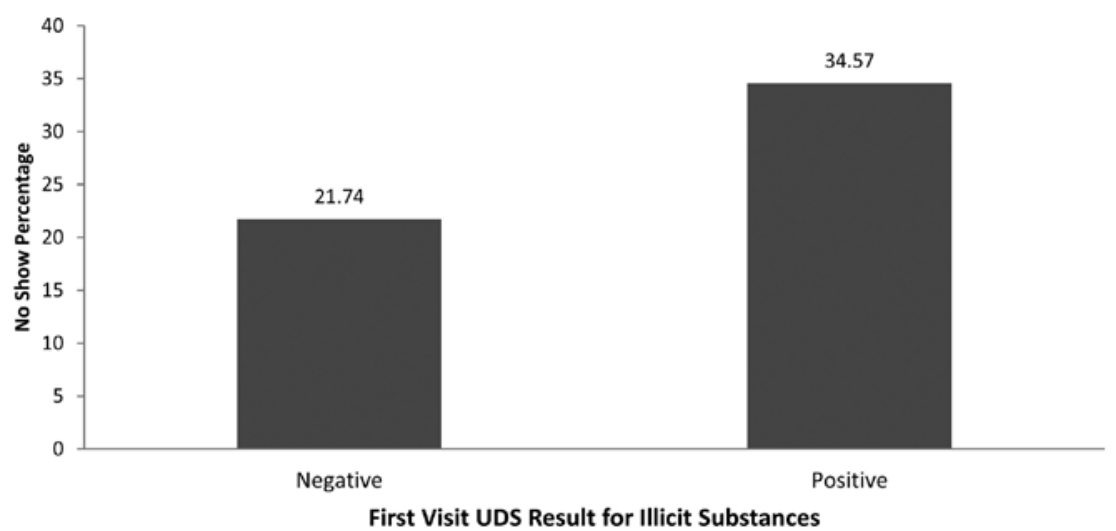

First Visit UDS Result for Illicit Substances 
Pain Physician: February 2016; 19:89-100

Table 6. Extended-Cox model effects on likelihood of dropout from therapy.

\begin{tabular}{|c|c|c|c|c|c|c|c|c|}
\hline Parameter & DF & $\begin{array}{l}\text { Parameter } \\
\text { Estimate }\end{array}$ & $\begin{array}{l}\text { Standard } \\
\text { Error }\end{array}$ & Chi-Square & $P$-value & $\begin{array}{c}\text { Hazard } \\
\text { Ratio }\end{array}$ & \multicolumn{2}{|c|}{$\begin{array}{c}\text { 95\% H.R Confidence } \\
\text { Limits }\end{array}$} \\
\hline \multicolumn{9}{|l|}{ Dropout3x } \\
\hline Age & 1 & -0.01429 & 0.00667 & 4.5915 & 0.0321 & 0.986 & 0.973 & 0.999 \\
\hline Gender & 1 & 0.06789 & 0.17389 & 0.1524 & 0.6962 & 1.07 & 0.761 & 1.505 \\
\hline UDS & 1 & 0.69454 & 0.13573 & 26.1834 & $<.0001$ & 2.003 & 1.535 & 2.613 \\
\hline OpioidRx & 1 & -0.51893 & 0.08636 & 36.1071 & $<.0001$ & 0.595 & 0.502 & 0.705 \\
\hline Psychiatry Referral & 1 & 0.00472 & 0.17289 & 0.0007 & 0.9782 & 1.005 & 0.716 & 1.41 \\
\hline Current Pain Score & 1 & 0.07655 & 0.0337 & 5.1583 & 0.0231 & 1.08 & 1.011 & 1.153 \\
\hline \multicolumn{9}{|l|}{ Dropout180 } \\
\hline Age & 1 & -0.01143 & 0.00754 & 2.2998 & 0.1294 & 0.989 & 0.974 & 1.003 \\
\hline Gender & 1 & 0.01749 & 0.19934 & 0.0077 & 0.9301 & 1.018 & 0.689 & 1.504 \\
\hline UDS & 1 & 0.75964 & 0.15076 & 25.3906 & $<.0001$ & 2.138 & 1.591 & 2.872 \\
\hline OpioidRx & 1 & -0.67744 & 0.1051 & 41.5481 & $<.0001$ & 0.508 & 0.413 & 0.624 \\
\hline Psychiatry Referral & 1 & -0.06111 & 0.20309 & 0.0905 & 0.7635 & 0.941 & 0.632 & 1.401 \\
\hline Current Pain Score & 1 & 0.05138 & 0.03903 & 1.7332 & 0.188 & 1.053 & 0.975 & 1.136 \\
\hline \multicolumn{9}{|l|}{ Dropout365 } \\
\hline Age & 1 & -0.01603 & 0.01165 & 1.893 & 0.1689 & 0.984 & 0.962 & 1.007 \\
\hline Gender & 1 & -0.34576 & 0.29522 & 1.3716 & 0.2415 & 0.708 & 0.397 & 1.262 \\
\hline UDS & 1 & 0.98006 & 0.24059 & 16.5943 & $<.0001$ & 2.665 & 1.663 & 4.27 \\
\hline OpioidRx & 1 & -0.73036 & 0.16528 & 19.5262 & $<.0001$ & 0.482 & 0.348 & 0.666 \\
\hline Psychiatry Referral & 1 & 0.39615 & 0.2628 & 2.2723 & 0.1317 & 1.486 & 0.888 & 2.487 \\
\hline Current Pain Score & 1 & 0.08487 & 0.06246 & 1.8464 & 0.1742 & 1.089 & 0.963 & 1.23 \\
\hline
\end{tabular}

We now provide a more detailed discussion of the results as it pertains to our original research question.

The first question in our research was whether screening in the first visit influences behavior subsequent to the visit, specifically no-show in the second visit. We found that receiving UDS in the first visit is associated with an increased likelihood of no-shows in the second visit. This is contrary to the expectation that the intent of monitoring is to increase, not decrease adherence. Given that keeping appointments is a key indicator of adherence, the finding that UDS early in the patient tenure is associated with increase in no-shows is at odds with the expectation that UDS ought to increase adherence.

Second, we found that patients who tested positive were more likely to be no-shows compared to those testing negative. This can be understood as follows: The UDS represents a signal of watchfulness on the part of the practice to the patient who is currently using illicit substances. If this is indeed the case, at least from the view of the clinic, the deterrent effect of UDS on patients currently using illicit substances is a desirable one.

However, the third, somewhat surprising finding is that even those who tested negative for illicit substances in the UDS were more likely to be no-shows compared to those who did not get tested. This raises concerns that the UDS administered early in the doctor-patient relationship might have an inadvertent impact on injuring patient expectations of trust. This is a concern that has been raised in the literature (17). In this regard, we should note that some of those testing negative for illicit substances may have clean samples on the day of testing but may in fact be using illicit substances. In our data, we found that of the first-test-negative patients who were tested a second time, $10.23 \%$ tested positive for illicit substances. Thus, while it is reasonable to be concerned that UDS early in the patient tenure may adversely impact the therapeutic alliance among those testing negative, it is important to take 
into consideration the possibility that one negative test does not provide the complete picture in regards to patient adherence with the opioid contract. The lack of completeness is also due to the retrospective nature of our data.

Finally, consistent with the above findings, we observed that UDS is also associated with the likelihood of dropping out from the therapy, suggesting a potential deterrent effect. In the present study, in most (but not all) instances, UDS was administered early in the patient tenure. This raises the question of whether UDS in the initial visit may be offending or otherwise straining the trust of the patients before the physician has had a chance to establish rapport, but that UDS later in the tenure may have a more salutary effect in regards to adherence.

Taken together the findings support the view that UDS may, in effect, be deterring people who are at high risk for abuse (as indicated by a positive test for illicit substances) from further engagement with the clinic. From the perspective of the clinic, UDS sends the signal that the practice is watchful, potentially deterring individuals with scope for misuse from the possibility of obtaining opioids. From a public health perspective, the implication of this effect is more complex; if the patients are disengaging from the clinic, where are they going? Is the illicit market place their next stop? Thus, while UDS may induce the problematic patients to go away from the clinic, the problem of opioid misuse may continue to persist since for these patients going out of the ambit of clinical care.
A related question is what can be done about dropouts and no-shows? Clearly, not testing is not an option. Jamison and colleagues (22) conducted a randomized control study of patients that either had a history of opioid misuse or were judged to be at risk thereof, and found that behavioral support through individual and group counseling, coupled with extensive monitoring significantly reduced the drug-misuse index score. Although this research does not speak to dropouts per se, it raises the possibility that consistent behavioral support for at risk patients might help reduce dropout even as they are monitored with UDS.

One of the important limitations of the present study is that it is a retrospective observational study rather than a randomized controlled experiment. We have tried to address some of the limitations of such observational studies by studying the effects in propensity score-matched datasets which account for differences in observed variables and through within-subjects analysis that controls for subject-specific observed/unobserved factors. Although these are accepted methods of addressing potential selection effects in observational studies, we cannot make causal claims in regards to the whether UDS alters no-shows and dropouts; that causal claim can only be tested in a prospective study involving random assignment of patients to UDS. In balance, we believe that the analysis presented here represents a first step in understanding the potential association between UDS and post-screening behavior, and that further research is needed to enhance our understanding of how monitoring influences behavior of patients considered for opioid therapy. 


\section{References}

1. Jha VM, Blum A. Abusive prescribing of controlled substances. N Engl] Med 2013; 369:2269-2270.

2. Volkow ND, McLellan TA. Curtailing diversion and abuse of opioid analgesics without jeopardizing pain treatment. JAMA 2011; 305:1346-1347.

3. Ives TJ, Chelminski PR, Hammett-Stabler CA, Malone RM, Perhac JS, Potisek NM, Shilliday BB, DeWalt DA, Pignone MP. Predictors of opioid misuse in patients with chronic pain: A prospective cohort study. BMC Health Serv Res 2006; 6:46.

4. Bohnert AS, Valenstein $M$, Bair MJ, Ganoczy D, McCarthy JF, Ilgen MA, Blow FC. Association between opioid prescribing patterns and opioid overdose-related deaths. JAMA 2011; 305:1315-1321.

5. Fishbain DA, Cole B, Lewis J, Rosomoff $H L$, Rosomoff RS. What percentage of chronic nonmalignant pain patients exposed to chronic opioid analgesic therapy develop abuse/addiction and/or aberrant drug-related behaviors? A structured evidence-based review. Pain Med 2008; 9:444-459.

6. Betses M, Brennan T. Abusive prescribing of controlled substances - a pharmacy view. N Engl J Med 2013:369: 989991.

7. Manchikanti L, Manchukonda R, Pampati V, Damron KS, Brandon D, Cash K, McManus C. Does random urine drug testing reduce illicit drug use in chronic pain patients receiving opioids? Pain Physician 2006; 9:123.

8. Chou R, Fanciullo GJ, Fine PG, Adler JA, Ballantyne JC, Davies P, Donovan MI,
Fishbain DA, Foley KM, Fudin J, Gilson AM, Kelter A, Mauskop A, O'Connor PG, Passik SD, Pasternak GW, Portenoy RK, Rich BA, Roberts RG, Todd KH, Miaskowski C. Clinical guidelines for the use of chronic opioid therapy in chronic noncancer pain. J Pain 2009; 10:113-130. el22.

9. Vadivelu N, L Chen I, Kodumudi V, Ortigosa E, Teresa Gudin M. The implications of urine drug testing in pain management. Curr Drug Safety 2010; 5:267270.

10. Kaye AD, Marshall ZJ, Lambert S, Trescot AM, Prabhakar A, Elhassan AO, Urman RD. Ethical Perspectives on Urine Drug Screening for Pain Physicians. Pain physician 2014:17: $\mathrm{E}_{559}-\mathrm{E}_{564}$

.11. Webster LR, Webster RM. Predicting aberrant behaviors in opioid-treated patients: Preliminary validation of the opioid risk tool. Pain Med 2005; 6:432-442.

12. Pollini RA, Banta-Green CJ, CuevasMota J, Metzner M, Teshale E, Garfein RS. Problematic use of prescriptiontype opioids prior to heroin use among young heroin injectors. Substance abuse and rehabilitation 2011; 2:173.

13. Starrels JL, Becker WC, Alford DP, Kapoor A, Williams AR, Turner BJ. Systematic review: Treatment agreements and urine drug testing to reduce opioid misuse in patients with chronic pain. Ann Intern Med 2010; 152:712-720.

14. Manchikanti L, Giordano J, Boswell MV, Fellows B, Manchukonda R, Pampati BV. Psychological factors as predictors of opioid abuse and illicit drug use in chronic pain patients. J Opioid Manag
2007; 5:8.

15. Turk DC, Swanson KS, Gatchel RJ. Predicting opioid misuse by chronic pain patients: A systematic review and literature synthesis. Clin J Pain 2008; 24:497-508 410.1097/AJP.109ob1013e31816b31070.

16. Chou R, Fanciullo GJ, Fine PG, Miaskowski C, Passik SD, Portenoy RK. Opioids for chronic noncancer pain: Prediction and identification of aberrant drugrelated behaviors: A review of the evidence for an American Pain Society and American Academy of Pain Medicine clinical practice guideline. J Pain 2009; 10:131-146. e135.

17. Katz N, Fanciullo GJ. Role of urine toxicology testing in the management of chronic opioid therapy. Clin J Pain 2002; 18:S76-S82.

19. Hagihara A, Hasegawa M, Abe T, Wakata Y, Nagata T, Nabeshima Y. Prehospital lactated ringer's solution treatment and survival in out-of-hospital cardiac arrest: A prospective cohort analysis. PLoS Med 2013; 10:e1001394.

19. Austin PC. A comparison of 12 algorithms for matching on the propensity score. Stat Med 2014; 33:1057-1069.

20. Coca-Perraillon M. Local and global optimal propensity score matching, SAS Global Forum, 2007, pp 1-9.

21. Thomas W. pp Propensity Score Matching Macro for SAS.

22. Jamison RN, Ross EL, Michna E, Chen LQ, Holcomb C, Wasan AD. Substance misuse treatment for high-risk chronic pain patients on opioid therapy: A randomized trial. Pain 2010; 150:390-400. 